\title{
Validity of BMI, hip and waist circumferences as surrogate measures of obesity in a cohort of Sri Lankan premenopausal women
}

\author{
J Nanayakkara ${ }^{1}$, S Lekamwasam ${ }^{2}$ \\ (Index words: central obesity, surrogate markers, Sri Lanka, women)
}

\begin{abstract}
Introduction BMI, hip and waist circumferences ( $\mathrm{HC}$ and WC) are being used as clinical surrogates of obesity. Unceratinities exist regarding the cut-off values, which are recommonded for Western countries.

Objectives We conducted a study to determine cut-off values for Sri Lankan women.

Methods Healthy premenopausal women $(n=128)$ aged 25 to 50 years were selected randomly, from local $\mathrm{MOH}$ area and stratified into four groups (32 in each) according to their BMI. Those who were pregnant, breast feeding, or on long-term medications were excluded. Body weight and height, hip circumference $(\mathrm{HC})$ and waist circumference (WC) were measured, using standard protocols. Lean and fat mass, were measured by DXA and percentage $\mathrm{FM}(\% \mathrm{FM})$ was calculated (FM/body weight $\mathrm{x} 100)$. Women with $\% \mathrm{FM}>30 \%$ were considered obese.

Results BMI moderately correlated $(r=0.41)$ with $\% \mathrm{FM}$ and BMI accounted for only $16 \%\left(r^{2}=0.16\right)$ of $\% F M$ variation. Regression equations were used to estimate the cut-off values that corresponded to \%FM of 30 . Those cut-off values for BMI, WC, and HC were $24.4 \mathrm{Kg} / \mathrm{m}^{2}, 92$ $\mathrm{cm}$, and $78 \mathrm{~cm}$, respectively.

Conclusion BMI, WC and $\mathrm{HC}$ values of $24 \mathrm{~kg} / \mathrm{m}^{2}, 92 \mathrm{~cm}$ and $78 \mathrm{~cm}$ can be considered appropriate cut-off values when detecting central obesity in premenopausal women.
\end{abstract}

Ceylon Medical Journal 2013; 58: 72-75

\section{Introduction}

Obesity is an emerging health problem, not only in the Western world, but also in South East Asia. It is a well recognised co-morbid factor linked to many health and social problems. Obesity is associated with insulin resistance, hyperinsulinaemia, dyslipidaemia, glucose intolerance and high blood pressure and contributes to morbidity and mortality related to non-communicable diseases, significantly.

Body mass index (BMI) is used as a clinical surrogate of central obesity. Currently Sri Lanka follows the standards adapted by the World Health Organization (WHO) in which BMI cut-off values of overweight and obesity are defined as $>25 \mathrm{Kg} / \mathrm{m}^{2}$ and $>30 \mathrm{~kg} / \mathrm{m}^{2}$, respectievly. The WHO developed this consensus by considering the 5th and 95th centiles of body weight and the mortality profile derived from cardiovascular moratility and morbodity data obtained perdominently from western countries [1]. Due to the high body fat content in Asians compared to Europeans, the direct applicability of these BMI values in Asian population is doubtful [2]. Furthermore, factors that are relevant to body compositiondietary habits and physical activity, have a geographical variation. BMI alone does not discriminate between fat mass and the fat-free mass in the body and its ability to predict the percentage fat mass accurately, is doubtful.

Apart from using epidemiological data to determine the BMI cut-off values, obesity can be defined based on the body fat content. In previous studies, percentage of body fat mass (BF\%) over $30 \%$ has been used to define obesity [2]. The 3rd National Health and Nutrition Examination Survey (NHANES III) found that BMI-defined obesity (BMI $>30$ ) was present in $21 \%$ of men and $31 \%$ of women in the USA. However, when BF\% was used 50\% of men and $62 \%$ of women were found to be obese. While BMI-defined obesity showed high specificity, the sensitivity was poor. BMI only identified $36 \%$ of the men and $49 \%$ of the women who had BF\%-defined obesity [3].

Sri Lanka does not have a large database of BMI and cardiovascular mortality and morbidity data to determine a suitable BMI threshold to define obesity. Hence, in this study we examined the applicability of currently used anthropometric cut-off values to define obesity and overweight in Sri Lanka and also attempted to define new thresholds to define obesity, based on the BF\% in the local population.

\section{Methods}

A cross-sectional study was conducted using a randomly selected group of 128 healthy premenopausal women aged 25-50 years. A community-based stratified sample was selected from female permanent residents in the Bope-Poddala MOH area. There are 18 Public Health Midwifery (PHM) areas in the Bope-Poddala division and

Departments of ${ }^{1}$ Pharmacology and ${ }^{2}$ Medicine, Faculty of Medicine, University of Ruhuna, Sri Lanka.

Correspondence: JN, e-mail: <nanajeevan@yahoo.com>. Received 11 October and revised version accepted 17 November 2012. Competing interests: none declared. 